\title{
Comportamiento de la urgencia ginecológica durante la pandemia de COVID-19
}

\section{Behavior of the gynecological emergency during the COVID-19 pandemic}

\section{Felipe Soto-Opitz ${ }^{1 *}$, Rafael Velasco ${ }^{3}$, Víctor Miranda ${ }^{2,4}$ y Sebastián Viguera ${ }^{2}$}

${ }^{1}$ Servicio de Obstetricia y Ginecología, Pontificia Universidad Católica; ${ }^{2}$ Servicio de Ginecología, Hospital Clínico, Pontificia Universidad Católica; ${ }^{3}$ Servicio de Medicina, Pontificia Universidad Católica; ${ }^{4}$ Departamento de Ginecología, Pontificia Universidad Católica. Santiago, Chile

\section{Resumen}

Introducción: La pandemia de COVID-19 ha comportado una disminución de la consulta ginecológica al servicio de urgencia. Objetivo: Caracterizar las consultas en el servicio de urgencia ginecológica, evaluando la cantidad de hospitalizaciones, la necesidad de intervención quirúrgica y los factores de morbilidad, entre otros, comparado a la situación con la previa a la pandemia. Método: Se realizó un estudio de cohorte no concurrente de pacientes hospitalizadas tras una consulta espontánea al servicio de urgencia ginecológica entre las semanas 12 y 28 de 2019 y 2020 en el Hospital Clínico de la Pontificia Universidad Católica, en Santiago (Chile). Se determinaron distintos factores de morbilidad: diagnóstico de ingreso, intervención quirúrgica, complicación operatoria, días de hospitalización, hemoglobina/hematocrito de ingreso y necesidad de transfusión de unidades sanguíneas. Resultados: Hubo 511 consultas al servicio de urgencia entre las semanas 12 y 28 del año 2019, en comparación con 196 el año 2020. En 2019 fueron hospitalizadas 103 mujeres, y en 2020 ingresaron 72 (odds ratio [OR]: 2.3). Disminuyó el ingreso por aborto retenido (24 vs. 12; $p=0.01$ ), mientras que aumentó el ingreso por metrorragia posmenopáusica ( 9 vs 22; $p=0.01)$. No hubo cambio en las intervenciones quirúrgicas realizadas $(86.4 \%$ vs. 84.7\%; $p=0.7$ ). Aumentaron los días de hospitalización (2.3 vs. 3.1; $p$ < 0.0001) y la necesidad de transfusión sanguínea (2 vs. 7; $p=0.02$; OR: 5.4; intervalo de confianza: 1.09-26). Conclusiones: La pandemia de COVID-19 provocó una disminución en la consulta espontánea por patología ginecológica al servicio de urgencia, provocando un aumento en la relación consultalingreso y una mayor morbilidad en las pacientes hospitalizadas, caracterizada por una mayor necesidad de transfusión sanguínea y un aumento de los días de hospitalización.

Palabras clave: Coronavirus. COVID-19. SARS-CoV-2. Unidad de emergencia. Ginecología. Hospitalización.

\section{Abstract}

Introduction: The COVID-19 pandemic has meant a decrease in gynecological visits to the emergency department. Objective: To characterize the attending in the gynecological emergency service, evaluating the amount of admissions, hospitalizations, need for surgical intervention, morbidity factors, among others, compared to the pre-pandemic situation. Method: A non-concurrent cohort study of hospitalized patients was carried out through a spontaneous attending to the gynecological emergency service between weeks 12 and 28 of 2019 and 2020 at the Hospital Clínico of the Pontificia Universidad Católica, in Santiago (Chile). Different morbidity factors were measured: admission diagnosis, surgical intervention, surgical complication, hospital stay, admission hemoglobin, and need for transfusion of blood units. Results: A total of 511

Disponible en internet: $27-10-2021$ Rev Chil Obstet Ginecol. 2021;86(4):347-352

www.rechog.com 0048-766X / @ 2021 Sociedad Chilena de Obstetricia y Ginecología. Publicado por Permanyer. Éste es un artículo open access bajo la licencia CC BY-NC-ND (https://creativecommons.org/licenses/by-nc-nd/4.0/).
} 
Rev Chil Obstet Ginecol. 2021;86(4)

visitors to the emergency service were registered between weeks 12 and 28 of 2019, compared to 196 in 2020 . In 2019 and 2020, 103 and 72 women were hospitalized respectively (odds ratio [OR]: 2.3). Admission for pregnancy loss decreased (24 vs. 12; $p=0.01$ ), while admission for postmenopausal bleeding increased (9 vs. 22; $p=0.01$ ). There was no change in the surgical interventions performed ( $86.4 \%$ vs. $84.7 \% ; p=0.7)$. The hospital stays increased $(2.3$ vs. $3.1 ; p<0.0001)$ and the need for blood transfusion (2 vs. $7 ; p=0.02 ;$ OR: 5.4; confidence interval: 1.09-26). Conclusions: The COVID-19 pandemic caused a decrease in spontaneous attending for gynecological pathology at the emergency service, causing an increase in the attend/admission ratio and greater morbidity in hospitalized patients characterized by a greater need for blood transfusion and an increase on the hospital stay.

Key words: Coronavirus. COVID-19. SARS-CoV-2. Emergency department. Gynecology. Hospital stay.

\section{Introducción}

La pandemia de COVID-19 ha puesto a prueba todos los sistemas de salud del mundo, en los cuales se ha vuelto especialmente importante el control de patologías respiratorias a cargo de los servicios de urgencia general y medicina interna. Sin embargo, también ha generado cambios y necesidad de adaptación en todo el sistema de salud, como en la atención de patologías no COVID-19 y enfermedades crónicas.

Una de las principales formas de enfrentar la pandemia por SARS-CoV-2 ha sido redestinar recursos y personal a la atención de esta patología, lo que ha generado la transformación de consultas presenciales en telemedicina y la reprogramación de cirugías electivas o no urgentes, entre otros cambios. Rápidamente, en el transcurso de la pandemia, distintas asociaciones hicieron recomendaciones para el manejo de patologías ambulatorias y suspensión de cirugías electivas con el fin de redestinar recursos de salud y personal hacia pacientes contagiados con SARS-CoV-2 $2^{1,2}$. Es así como los servicios de urgencia tuvieron que preparar sus equipos para la atención de patologías graves, al mismo tiempo que realizar educación a la población con el objetivo de recibir consultas de urgencia seleccionadas.

El primer caso de COVID-19 en Chile fue pesquisado el día 3 de marzo de 2020 (semana 10 del año), el decreto de Estado de Excepción Constitucional de Catástrofe se inició el día 18 de marzo de 2020 (semana 12 del año), mientras que las primeras cuarentenas fueron decretadas el 25 de marzo de 2020 (semana 13 del año). Una de las preocupaciones iniciales fue la eventual caída de las consultas de patología no COVID-19, debido a la dificultad de acceso a los servicios de emergencia, producto del colapso sanitario o del miedo a enfermar por parte de la población al acudir a establecimientos de salud. Desde la semana 12 del año ya se observó en Chile una disminución de atenciones por enfermedades cuyo desenlace depende del tiempo, como por ejemplo accidentes cerebrovasculares, alertando así una posible demora en la atención que podría llevar a un aumento de las complicaciones asociadas ${ }^{3}$.

Entre las semanas 12 y 28 se realizaron 252,841 consultas de atención de urgencia en la Región Metropolitana de Chile, lo cual dista del año 2019, cuando entre las mismas semanas se cuantificaron 626,414 consultas. Es decir, hubo una disminución del $59.6 \%$ en las consultas de atención en urgencias con respecto al año anterior (Fig. 1).

Un estudio español analizó las consecuencias en la atención de cirugías de urgencia comparando pacientes atendidos durante la pandemia con el mismo periodo del año anterior, y se encontró una disminución en las consultas de urgencia, un aumento en los tiempos entre el inicio de los síntomas y la consulta a la urgencia (44 vs. 71 horas) y un aumento de la morbili$\mathrm{dad}^{5}$. En Italia se evidenció en un servicio de ginecología una disminución en el total de consultas, siendo el principal motivo el dolor pélvico; así mismo, se encontró un aumento en la tasa de hospitalización y en la necesidad de intervención quirúrgica en comparación con el periodo previo a la pandemia ${ }^{6}$.

La demora en obtener atención y resolución de patologías ginecológicas puede llevar al aumento de la morbilidad secundaria y la necesidad de resolución quirúrgica, como es el caso del embarazo ectópico y el sangrado uterino anormal ${ }^{7-9}$.

Hasta el momento no existen estudios en población chilena sobre las consecuencias en pacientes con patología ginecológica producto de la atención durante la pandemia.

Nuestro objetivo fue llevar a cabo un estudio analítico de cohorte no concurrente para analizar las características y la evolución de las pacientes hospitalizadas por causa ginecológica a través del servicio de urgencia de ginecología en el Hospital Clínico de la Pontificia Universidad Católica, de la Red de Salud UC-Christus, entre las semanas 12 y 28 del año 2020, momento en que se llegó al pico de COVID-19, y compararlas con 


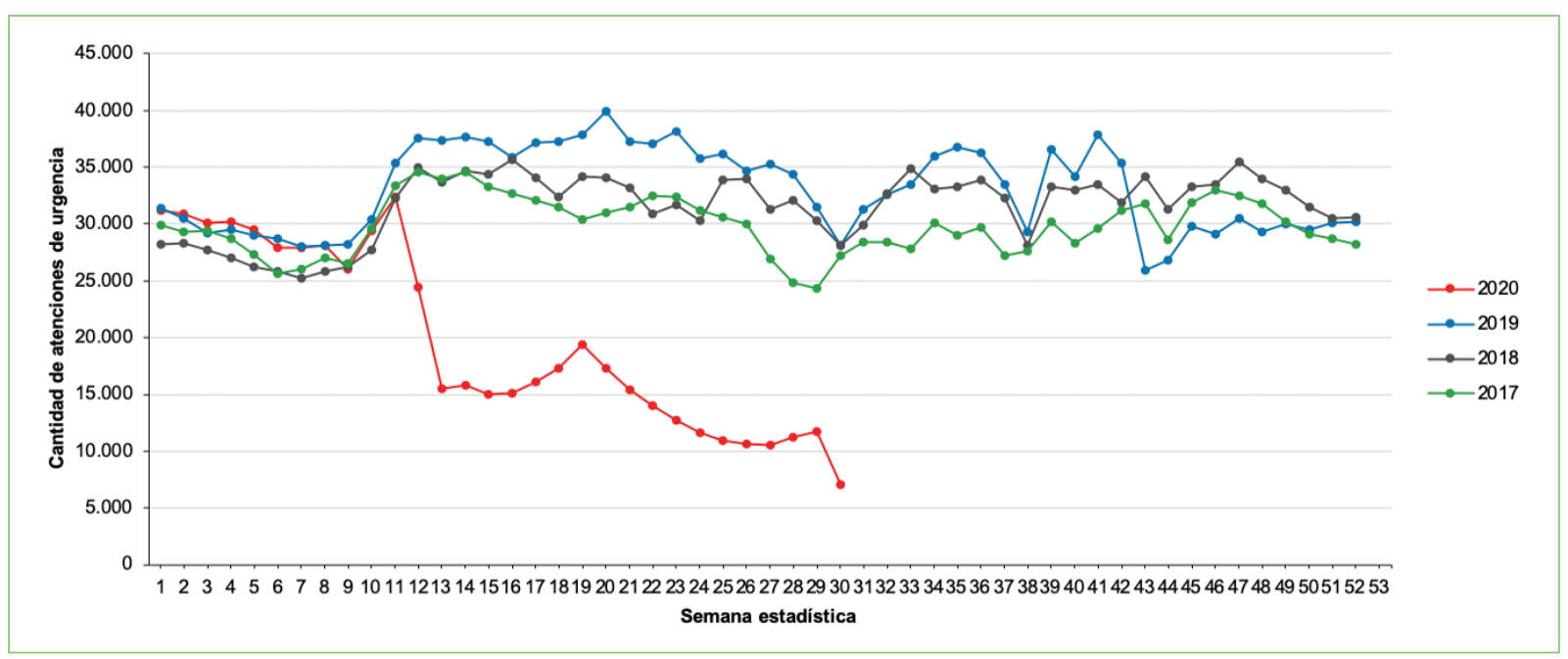

Figura 1. Comparación de cantidad de consultas en Servicios de Urgencia en la Región Metropolitana entre los años 2017-2020 según semana estadística.

las de las pacientes ingresadas en el mismo establecimiento durante las semanas 12 y 28 del año 2019.

En este estudio esperamos identificar un menor número de consultas ginecológicas, un aumento de cirugías de urgencia realizadas y un mayor grado de complicaciones en estas pacientes, las cuales podrían explicarse por el contexto de COVID-19 en el país. Con los resultados podremos caracterizar la evolución de las pacientes con patología ginecológica en nuestra institución durante la pandemia y predecir el comportamiento de esta en futuros rebrotes.

\section{Método}

Se realizó un estudio analítico de cohorte no concurrente, reclutando pacientes hospitalizadas por causa ginecológica a través del servicio de urgencia ginecológica del Hospital Clínico de la Pontificia Universidad Católica, en Santiago (Chile), entre las semanas 12 y 28 de los años 2019 y 2020, las cuales corresponden a los periodos entre el 18 de marzo y el 14 de julio de 2019 y entre el 16 de marzo y el 12 de julio de 2020. Se incluyeron en total 103 pacientes hospitalizadas en el periodo de 2019 y 72 pacientes hospitalizadas en el periodo de 2020. Se excluyeron las pacientes cursando un embarazo de más de 12 semanas.

Para responder la pregunta “¿Produjo la pandemia un cambio en la atención de urgencia de patologías ginecológicas?" se presenta como objetivo primario comparar la tasa de hospitalización de urgencia por causa ginecológica durante la época de pandemia con la tasa del año anterior (prepandemia). Como objetivo secundario se busca caracterizar a las pacientes que consultaron en la urgencia de ginecología durante la época de pandemia, calcular y comparar la cantidad de cirugías de urgencia realizadas en estos periodos, y describir variables de morbilidad (días de hospitalización, necesidad de resolución quirúrgica, tipo de cirugía realizada, vía de cirugía, hemoglobina y hematocrito de ingreso, necesidad de transfusión, número de unidades de glóbulos rojos transfundidos, complicación quirúrgicas o posoperatorias, necesidad de rehospitalización y necesidad de reintervención) durante la hospitalización de las pacientes en estos periodos.

La recolección de datos se hizo en el registro de hospitalizaciones en red y por revisión de la ficha clínica y los protocolos operatorios de cada paciente.

Para el análisis estadístico se utilizó el software estadístico SAS (SAS Institute, Cary, NC). Todos los datos fueron analizados con la prueba de ji al cuadrado para datos categóricos, la prueba $t$ de Student para datos paramétricos continuos y la prueba de Wilcoxon para datos ordinales o continuos no paramétricos.

Para acceder a la ficha clínica de cada paciente se contó con la autorización del Comité Ético Científico de

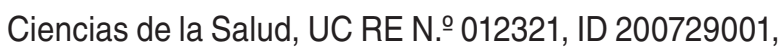
27 de agosto de 2020 .

\section{Resultados}

Durante el año 2019, entre las semanas 12 y 28, hubo 511 consultas al servicio de urgencia por causa ginecológica, sin contar pacientes cursando un embarazo de más de 12 semanas, mientras que en el año 
2020, durante el mismo periodo, se realizaron 196 consultas al servicio de urgencia, lo que supone una disminución del $61 \%$ en la cantidad de consultas a la unidad.

Del total de pacientes que consultaron, 103 requirieron hospitalización el año 2019, mientras que el año 2020 fueron 72. Se evidencia una relación consulta/ ingreso de 0.20 y 0.37 para los años 2019 y 2020, respectivamente (odds ratio [OR]: 2.3), lo que significa que durante el periodo de 2019 se hospitalizó a una de cada cinco pacientes que consultaron al servicio de urgencias y en el periodo de 2020 se ingresó a una de cada tres.

Se analizaron las siguientes variables paramétricas y no paramétricas: edad, motivo de consulta, diagnóstico de ingreso, intervención quirúrgica durante la hospitalización, tipo de cirugía y vía quirúrgica, hallazgo de hemoperitoneo durante la cirugía, uso de antibióticos, días de hospitalización, necesidad de reintervención, hemoglobina y hematocrito de ingreso, y necesidad de transfusión de unidades sanguíneas (Tabla 1).

\section{Discusión}

Durante la pandemia de COVID-19 se ha generado un cambio en la atención y la resolución de patologías no COVID-19 en todo el mundo, con una disminución de la consulta de emergencia de alrededor de un $60 \% 5,6,10,11$.

En este estudio se evidencia la disminución de consultas al servicio de urgencias ginecológicas de nuestro centro, con una disminución del $61 \%$ del total de consultas, asociado a un aumento en la razón de ingreso a través del servicio de urgencia en comparación con el año previo, con una OR de 2.3 para el riesgo de requerir hospitalización al consultar al servicio de urgencia, similar a lo visto en otros estudios ${ }^{11}$. Esto puede tener varias interpretaciones, así como un aumento de la complejidad de los casos ginecológicos que consultaron al servicio de urgencia durante la pandemia, como también una disminución en el motivo de consulta que no corresponde a una real urgencia, haciendo un correcto uso de los recursos.

Los estudios describen que el cambio en el modelo de atención ha generado que patologías como el embarazo ectópico hayan presentado un aumento en la necesidad de resolución quirúrgica, así como que también se haya producido un aumento de la presentación de hemoperitoneo ${ }^{7,8}$. En nuestro centro no hubo una diferencia significativa en el ingreso por embarazo ectópico complicado, pero sí en la patología relacionada con el sangrado uterino anormal.

El principal motivo de consulta de los grupos estudiados no varió, siendo en ambos el sangrado uterino anormal (31 vs. 33). Al estudiar el diagnóstico de ingreso, en el año 2019 el más frecuente fue el aborto retenido, mientras que en el año 2020 fue la metrorragia de la posmenopausia. Lo anterior puede ser interpretado como una decisión de la mujer durante el año 2020 , frente a la necesidad de intervención quirúrgica activa con el diagnóstico de aborto retenido en comparación con el manejo expectante para evitar el contacto e ingreso a una unidad hospitalaria con el riesgo de infección por SARS-CoV 2. Distinto es el caso de la metrorragia posmenopáusica, una patología que normalmente se resuelve de forma ambulatoria tanto en estudio como en tratamiento, lo cual, en vista del aumento en la necesidad de tratamiento intravenoso 0 resolución quirúrgica, pudo suponer un incremento en la necesidad de hospitalización.

El manejo del sangrado uterino anormal es variado y requiere un adecuado estudio para un correcto diagnóstico. Las guías internacionales promueven un mayor seguimiento a través de telemedicina para interpretar correctamente la urgencia y realizar un manejo ambulatorio con hormonoterapia para disminuir el ingreso hospitalario por esta causa?

No se encontró diferencia en la necesidad de intervención quirúrgica en las pacientes hospitalizadas durante 2019 y 2020 (86.4\% y 84.7\%; p = 0.7), pero sí en la cirugía específica realizada en cada periodo. Durante el año 2019 se realizaron 46 legrados uterinos de urgencia, mientras que en el año 2020 solo se realizaron 27. Esto se explica por la disminución de ingresos por aborto durante el último periodo. En el año 2020 aumenta la cantidad de histerectomías y de resectoscopias en comparación con el año previo. Estas intervenciones quirúrgicas habitualmente son realizadas de forma electiva y programada en pacientes no complicadas, pero debido a la disminución del control ambulatorio y a la suspensión de procedimientos electivos hubo que realizarlas en hospitalizaciones de urgencia.

El cambio en la modalidad de atención también refleja un mayor grado de complicaciones o eventos adversos en estas pacientes. Esto se confirma por un aumento de la presencia de hemoperitoneo, de seis casos en el periodo de 2019 a diez en el de 2020 ( $p=0.068$; OR: 2.6; intervalo de confianza [IC]: 0.97.5); un aumento en los días de hospitalización de 2.3 (desviación estándar [DE]: 0.79) a 3.1 (DE: 2.2) 
Tabla 1. Análisis de las variables según el año de atención

\begin{tabular}{|c|c|c|c|}
\hline Variable & $\begin{array}{c}2019 \\
(n=103)\end{array}$ & $\begin{array}{c}2020 \\
(n=72)\end{array}$ & p \\
\hline Edad, años & $\begin{array}{c}36.1 \\
\text { (DE: } 12.7)\end{array}$ & $\begin{array}{c}37.1 \\
\text { (DE: 12.3) }\end{array}$ & 0.74 \\
\hline $\begin{array}{l}\text { Motivo de consulta } \\
\text { Sangrado uterino } \\
\text { anormal } \\
\text { Dolor pelviano } \\
\text { Dolor abdominal } \\
\text { Aborto } \\
\text { Flujo } \\
\text { Control posoperatorio } \\
\text { Dolor mamario }\end{array}$ & $\begin{array}{l}31 \\
16 \\
23 \\
23 \\
3 \\
5 \\
2\end{array}$ & $\begin{array}{l}33 \\
10 \\
19 \\
9 \\
0 \\
1 \\
0\end{array}$ & 0.1 \\
\hline \multicolumn{4}{|l|}{ Diagnóstico de ingreso } \\
\hline $\begin{array}{l}\text { Aborto retenido } \\
\text { Aborto incompleto } \\
\text { Mioma uterino } \\
\text { Endometriosis } \\
\text { Metrorragia } \\
\text { posmenopáusica }\end{array}$ & $\begin{array}{c}24 \\
17 \\
2 \\
1 \\
9\end{array}$ & $\begin{array}{c}12 \\
6 \\
6 \\
0 \\
22 \\
\end{array}$ & $\begin{array}{c}0.01 \\
0.8 \\
0.57 \\
0.58 \\
0.01 \\
\end{array}$ \\
\hline $\begin{array}{l}\text { Proceso inflamatorio } \\
\text { pelviano } \\
\text { Tumor anexial o quiste } \\
\text { ovárico }\end{array}$ & $\begin{array}{c}1 \\
18\end{array}$ & $\begin{array}{l}2 \\
8\end{array}$ & $\begin{array}{l}0.57 \\
0.68\end{array}$ \\
\hline $\begin{array}{l}\text { Embarazo ectópico } \\
\text { complicado }\end{array}$ & 3 & 4 & 0.4 \\
\hline $\begin{array}{l}\text { Embarazo ectópico no } \\
\text { complicado }\end{array}$ & 5 & 1 & 0.57 \\
\hline Absceso de Bartholino & 9 & 5 & 0.1 \\
\hline Pólipo & 1 & 1 & 0.57 \\
\hline $\begin{array}{l}\text { Complicación } \\
\text { posoperatoria }\end{array}$ & 4 & 1 & 0.5 \\
\hline Otros & 9 & 4 & 0.3 \\
\hline \multicolumn{4}{|l|}{ Cirugía } \\
\hline Legrado & $\begin{array}{c}89 \\
(86.4 \%)\end{array}$ & $\begin{array}{c}61 \\
(84.7 \%)\end{array}$ & 0.7 \\
\hline Histerectomía & 46 & 27 & \\
\hline Resectoscopia & 2 & 6 & \\
\hline Drenaje de absceso & 2 & 7 & \\
\hline Salpingectomía & 9 & 7 & \\
\hline $\begin{array}{l}\text { Quistectomía o } \\
\text { anexectomía }\end{array}$ & 5 & 5 & \\
\hline Laparotomía & 15 & 5 & \\
\hline exploradora & 1 & 1 & \\
\hline $\begin{array}{l}\text { Laparoscopia } \\
\text { exploradora }\end{array}$ & 1 & 0 & \\
\hline Otra & 8 & 3 & \\
\hline \multicolumn{4}{|l|}{ Vía quirúrgica } \\
\hline Vaginal & 62 & 35 & 0.03 \\
\hline Laparoscópica & 19 & 10 & \\
\hline $\begin{array}{l}\text { Histeroscópica } \\
\text { Laparotomía }\end{array}$ & $\begin{array}{l}2 \\
6\end{array}$ & $\begin{array}{l}7 \\
9\end{array}$ & \\
\hline Hemoperitoneo & 6 & 10 & 0.068 \\
\hline Uso de antibióticos & 14 & 11 & 0.75 \\
\hline Días de hospitalización & $\begin{array}{c}2.3 \\
\text { (DE: } 0.79)\end{array}$ & $\begin{array}{c}3.1 \\
\text { DE: } 2.2)\end{array}$ & $<0.0001$ \\
\hline $\begin{array}{l}\text { Necesidad de } \\
\text { reintervención }\end{array}$ & 1 & 3 & 0.1 \\
\hline Hemoglobina (g/dl) & $\begin{array}{c}12.5 \\
\text { (DE: } 1.96)\end{array}$ & $\begin{array}{c}11.4 \\
\text { (DE: } 2.35)\end{array}$ & 0.12 \\
\hline Hematocrito (\%) & $\begin{array}{c}36.8 \\
\text { (DE: } 5.2)\end{array}$ & $\begin{array}{c}34.3 \\
\text { (DE: } 6.4)\end{array}$ & 0.069 \\
\hline $\begin{array}{l}\text { Necesidad de transfusión } \\
\text { de unidades sanguíneas }\end{array}$ & 2 & 7 & 0.02 \\
\hline
\end{tabular}

( $p<0.0001$ ); una disminución en el hematocrito al ingreso del $36.8 \%$ (DE: 5.2 ) al $34.3 \%$ (DE: 6.4) ( $p=$ $0.069)$; y un aumento en la necesidad de transfusión de unidades de glóbulos rojos de 2 a 7 ( $p=0.02$; OR: 5.4; IC: 1.09-26).

\section{Conclusiones}

La pandemia de COVID-19 nos ha demostrado durante el año 2020 lo importante que es disponer de un sistema de salud organizado que pueda abarcar las necesidades de su población en momentos de emergencia sanitaria. Es así como distintos gobiernos y organizaciones de salud internacionales redactaron recomendaciones sobre el manejo de patologías no respiratorias en tiempos de pandemia ${ }^{1,2}$.

El presente estudio describe los cambios ocurridos en un servicio de urgencia durante la pandemia de COVID-19, observándose una disminución de las consultas y un aumento de los casos con complicaciones. Si bien este estudio fue realizado en una institución privada, por lo que no se puede generalizar al total de la población, resulta de utilidad para que distintas instituciones revisen el comportamiento que tuvieron sus atenciones de urgencias durante este periodo y las diferencias en comparación con periodos previos. Al conocer estos cambios se podrá planificar de mejor manera el flujo de atención, permitiendo consecuentemente estar preparados ante eventuales rebrotes.

Es posible proponer, con los resultados obtenidos, un continuo seguimiento a través de un control extrahospitalario, como telemedicina o llamada telefónica, para pacientes con patologías crónicas que pueden agravarse. Además, una mejor selección al programar cirugías para patologías cuyo desenlace depende del tiempo, así como la resolución de cuadros de sangrado uterino anormal. Es importante destacar que la resolución de cuadros ginecológicos que no representan un riesgo vital inminente no debe entorpecer la correcta gestión de camas para pacientes con COVID-19.

\section{Financiamiento}

Los autores declaran no tener financiamiento para la realización de este estudio.

\section{Conflicto de intereses}

Los autores declaran no tener conflicto de intereses para la realización de este estudio. 


\section{Responsabilidades éticas}

Protección de personas y animales. Los autores declaran que para esta investigación no se han realizado experimentos en seres humanos ni en animales.

Confidencialidad de los datos. Los autores declaran que han seguido los protocolos de su centro de trabajo sobre la publicación de datos de pacientes.

Derecho a la privacidad y consentimiento informado. Los autores declaran que en este artículo no aparecen datos de pacientes.

\section{Bibliografía}

1. FIGO statement. Elective surgery and COVID-19, 30 de marzo de 2020 Disponible en https://www.figo.org/elective-surgery-and-covid-19-april2020-guidance. Consultado 01 Julio 2020.

2. SOCHOG, Declaración cirugía en periodo COVID19, 2 de abril de 2020 Disponible en https://sochog.cl/archivos/12984. Consultado 01 Julio 2020.
3. Toro L, Parra A, Alvo M. Epidemia de COVID-19 en Chile: impacto en atenciones de servicios de urgencia y patologías específicas. Rev Med Chile. 2020;148:558-60.

4. Ministerio de Salud. Indicadores básicos de Salud. Departamento de Estadísticas e Información en Salud, DEIS. Disponible en https://deis. minsal.cl/. Consultado 01 Agosto 2020.

5. Cano-Valderrama O, Morales X, Ferrigni CJ, Martín-Antona E, Turrado V, García A, et al. Acute care surgery during the COVID-19 pandemic in Spain: changes in volume, causes and complications. A multicentre retrospective cohort study. Int J Surg. 2020;80:157-61.

6. Grandi G, Del Savio MC, Caroli M, Capobianco G, Dessole F, Tupponi G et al. The impact of COVID-19 lockdown on admission to gynecological emergency departments: results from a multicenter Italian study. Int J Gynaecol Obstet. 2020;151:39-42.

7. Toma HV, Bank TC, Hoffman MK. Care for women with ectopic pregnancies during the coronavirus disease 2019 (COVID-19) pandemic. Obstet Gynecol. 2021;137:1041-2.

8. Dvash S, Cuckle H, Smorgick N, Vaknin Z, Padoa A, Maymon R. Increase rate of ruptured tubal ectopic pregnancy during the COVID-19 pandemic. Eur J Obstet Gynecol Reprod Biol. 2021;259:95-9.

9. Winters U, Ghosh M, Shelleh A. Heavy menstrual bleeding management during the Covid pandemic. Obstet Gynaecol Reprod Med. 2021;31:53-8.

10. Spurlin EE, Han ES, Silver ER, May BL, Tatonetti NP, Ingram MA, et al. Where have all the emergencies gone? The impact of the COVID-19 pandemic on obstetric and gynecologic procedures and consults at a New York City Hospital. J Minim Invasive Gynecol. 2021; 28:1411-9.e1.

11. Salsi G, Seidenari A, Diglio J, Bellussi F, Pilu G, Bellussi F. Obstetrics and gynecology emergency services during the coronavirus disease 2019 pandemic. Am J Obstet Gynecol MFM. 2020;2:100214. 